\title{
Antioxidant and Antimicrobial Properties of Cistus Ladanifer
}

\author{
Merve Deniz KÖSE ${ }^{1}$, Buse Nur TEKİN ${ }^{1}$, Oguz BAYRAKTAR ${ }^{1,2^{*}}$, Emre Taylan \\ DUMAN $^{2}$, Yücel BAŞPINAR ${ }^{3}$
}

${ }^{1}$ Department of Chemical Engineering, Ege University, Bornova, Izmir, Turkey

${ }^{2}$ NPRO Consult. Nat. Prod. Biotech. Ltd. Co., Ege Technology Development Zone, Bornova, Izmir, Turkey

${ }^{3}$ Department of Pharmaceutical Biotechnology, Ege University, Bornova, Izmir, Turkey

Received: 03 May 2017 - Revised: 13 September 2017 - Accepted: 03 November 2017

\begin{abstract}
Different parts of Cistus species are traditionally used in folk medicine as a remedy for several microbial disorders and infections. At the beginning of the study, crude extract of the Cistus leaves was obtained to determine its antioxidant and antimicrobial activities. Then volatile compounds were extracted using hydrodistillation and hexane. The Cistus oil was obtained by hydrodistillation of fresh material, using leaves in an altered Clevengertype device. Aqueous extract of Cistus leaves was neutralized to precipitate gums. The yield of gum was determined as $1 \%$. Resinoid was obtained after ethanol extraction of gum. Antibacterial activities of extract were determined using disc diffusion and micro-dilution assays against gram-positive and gram-negative bacteria. The extract of Cistus leaves tested and exhibited antibacterial activities by inhibiting one or more microorganisms. The tested plant extract was more active against gram-positive bacteria compared with gram-negative bacteria. Total phenol content of the extract was determined with Folin-ciocalteu method. Total phenolic content of Cistus extract was $520 \pm 15 \mathrm{mg}$ Gallic Acid Equivalent (GAE)/g extract. The water soluble (ACW) and lipid soluble (ACL) antioxidant capacities of the extract were also determined. ACW and ACL antioxidant capacities of Cistus extract were found as $650 \pm 80 \mu \mathrm{g}$ Ascorbic acid/ mg extract and $540 \pm 30 \mu \mathrm{g}$ Trolox Equivalent/ mg extract, respectively. In this study, there is a clear relationship between the total antioxidant and phenol content analysis results and antibacterial activities. Hereby, bioactive natural compounds present in Cistus species can be used as natural raw material in some related industrial applications.
\end{abstract}

Keywords: Cistus genus, Antibacterial activity, Antioxidant capacity, Labdanum

\section{INTRODUCTION}

There is an increasing interest in using medicinal and aromatic plants as natural sources in pharmaceutical, food and cosmetic industries all over the world. Bioactive compounds having antioxidant and antimicrobial activities isolated from medicinal plants had potential to be used in different industries as botanical drugs, dietary supplements, functional foods and food packaging etc.

Cistus genus is a plant traditionally used in folk medicine as a remedy for several microbial disorders and infections. The use of chemical products not only in the pharmaceutical field but also in food, cosmetic and hygiene industries are increasingly replaced by the use of antimicrobial agents derived from plants [1]. These naturally occurring compounds are

${ }^{*}$ Corresponding Author E-mail: oguz.bayraktar@ege.edu.tr 
considered a therapeutic alternative to the use of synthetic antibiotics, entailing a low risk of microbial resistance occurrence [2].

Due to their disease treatment features by eliminating or killing the infecting microorganisms, antimicrobial agents are the important chemicals that are widely used in modern medical practice. There are a various number of antimicrobial agents currently available. For the selection of a particular antimicrobial agent, its selective toxicity must be evaluated. Desired antimicrobial agent should exhibit greater toxicity to the infecting pathogens than to the host organism [3].

Turkey has one of the greatest floras in Europe due to its various numbers of plants also including many endemics [4]. The main purpose of this study was to determine the antioxidant and antimicrobial activities of extracts prepared from the leaves of Cistus sp. grown in Urla/ İzmir. Antioxidant capacity is the most important and widely used parameter to characterize and determine the quality of the various plant materials such as fruits, vegetables, wine, teas, oils and etc. Especially in the recent years, antioxidants especially from natural sources are becoming more and more convincing for fighting against oxidative stress damages [5]. Two different components, concrete and absolute can be prepared from Cistus sp.

Highly concentrated aromatic materials like concretes and absolutes can be extracted from Cistus plants. The multi-step process includes the first step extracting the aromatic oil from the plant material with a solvent such as hexane or heptane. Then the removal of hexane results in a waxy substance called concrete. This semi-solid to solid highly fragrant material contains a lot of pigments and waxes. They are soluble in both oil and alcohol.

The aromatic oils can be extracted from the concrete and then separated from most of the plant waxes and non-aromatic material with ethyl alcohol. With the removal of ethyl alcohol, the remaining substance is called an absolute. The absolute, which is highly regarded in natural perfumery, is the most concentrated form of natural fragrance. Even after removal with ethyl alcohol, absolutes still contain some of the waxes and pigments along with other constituents from the plant. However, they are mostly comprised of the concentrated aromatic oil.

There difference between absolutes and essential oils are different form each other such that essential oils do not contain waxes, are much lighter in color or have no color, and have a lighter aroma. Typically essential oils are used in skincare and for therapeutic purposes, while absolute and concretes are for natural perfumery. Through steam distillation of the branch, labdanum essence can be obtained. The resinoid is obtained through extraction, using volatile solvents. Products labeled as "Labdanum" are obtained by processing the Cistus resinous exudate, and include labdanum resinoid, labdanum absolute, and labdasur.

The main part responsible for the antimicrobial capacity of Cistus plants seems to be the volatile fraction containing essentially terpenes, although relevant activity has been also reported for the polyphenolic fraction [6]. Our results revealed the presence of volatile components in Cistus sp. Therefore it is possible to conclude that resin part of Cistus sp. has an antimicrobial activity against selected microorganisms. In this study gum resinoids, essential oil of labdanum resin crude extract of leaves was successfully prepared. Antioxidant and antimicrobial properties of extract were investigated. The resinoid was characterized using FTIR. The essential oil from labdanum resin was analyzed using Gas Chromatography.

\section{MATERIAL and METHODS}

\subsection{Materials}

Plant material (Cistus ladenifer) used in this study were collected from Urla- İzmir in September. 


\subsubsection{Chemicals}

For the assays, DMSO (Dimethyl sulfoxide-99.5\%) and Gallic acid were purchased from Amersco and Merck, respectively. Methanol (99.7\%) and ethanol absolute-chromasol (99.8\%) were obtained from Riedel. Folin-ciocalteu reagent and sodium carbonate anhydrous $(99.5 \%)$ were respectively obtained from Sigma and Fluka. Various growth medias (broth and agar) were used to ensure the cultivation of the microorganisms. Bacto Agar (214010) was obtained from Sinerji, Nutrient Agar (70116) and Nutrient Broth (70122) were purchased from Fluka. Mueller-Hinton Broth (A3751) was purchased from Applichem. To dilute the microbial cultures Bacto Peptone (211677) was used and obtained from Sinerji. Gentamicin and penicillin were used for comparison to evaluate the antimicrobial activities of plant extracts in microdilution assays. Gentamicin (15710) was purchased from Invitrogen and penicillin (Iecilline flakon $400.000 \mathrm{IU})$ was obtained from a pharmacy. Besides, penicillin G (CTOO43B), gentamicin (CTOO24B), and vancomycin (CTOO58B) were the antibiotic discs used in the disc diffusion assays and purchased from OXOID. Glycerol (15524) was used to prepare the stock cultures and store them in $-80^{\circ} \mathrm{C}$ (Revco). Finally, INT (Iodonitrotetrazolium chloride) was used as a dye in 96 well plates for visual identification of MICs (Minimum Inhibition Concentrations).

\subsubsection{Instruments and Equipment}

In order to evaluate the MIC values, microdilution tests were carried out; with an instrument called Varioskan (Thermo, microplate reader) was used. Microtiter 96 Well plates were obtained from Thermo. These sterile microplates have clear, flat bottom and polystyrene properties. To determine the antioxidant capacities of extracts Photochem (Analytik Jena) instrument was used and for total phenol determination tests UV spectrophotometer (Perkin Elmer) were used.

\subsection{Methods}

\subsubsection{Extraction Methods}

\subsubsection{Preparation of Gum}

The leaves of $C$. ladanifer $\left(20 \mathrm{~g}\right.$ ) were submerged into $0.1 \mathrm{M} \mathrm{NaHCO}_{3}$ solution for 10 min and kept at $60-70^{\circ} \mathrm{C}$ with $\mathrm{pH} 8.4$. The solution was filtered to remove the leaves. The filtered solutions were neutralized to $\mathrm{pH} 7$ with $0.2 \mathrm{M} \mathrm{HCl}$ solution.

\subsubsection{Preparation of Labdanum Resinoid}

$1 \mathrm{~g}$ of the gum was extracted with $4 \mathrm{~mL}$ of ethanol. The extract was cooled down and then filtered. Remaining was resinoid of labdanum.

\subsubsection{Preparation of Plant Extract}

C. ladanifer leaves were ground and extractions were performed at a solid to solvent ratio of 1:20 in $70 \%$ aqueous ethanol solutions for 24 hours. After the extraction ethanol was removed by using rotary evaporator and the remaining aqueous part was dried by using lyophilization to obtain the crude extract of Cistus leaves.

\subsubsection{Determination of Relative Antioxidant Activities of Cistus Extract}

PCL (Photochemiluminescence) assays [7] were carried out using PHOTOCHEM device (Analytik Jena AG (Jena Germany). To determine the relative antioxidant activities of plant extracts, PCL (Photochemiluminescence) method was used. By using a single system on photochem antioxidant capacities of water soluble (ACW) and antioxidant capacity of lipid soluble (ACL) compounds were specified. Total phenol content of the extract was determined 
with Follin-Ciocalteu method [8] and results were given in terms of mg Gallic acid Equivalent (GAE)/g extract.

\subsubsection{Determination of the Relative Antibacterial Activities of Plant Extracts}

\subsubsection{Disc Diffusion Assays}

In order to test the antibacterial activities of the extract, sterile filter discs impregnated with the extract solution were placed on agar plates containing the microorganisms.

The effectiveness of extract as an antimicrobial agent was shown by the presence of growth inhibition zones. The zones of inhibition appeared as clear areas surrounding the disc from which the substances with antimicrobial activity diffused. This zone was measured and recorded in $\mathrm{mm}$.

In the preliminary disc diffusion assays, three bacteria species were chosen to determine the antimicrobial activity of the extract. E. coli (NRRL B- 3008), Bacillus subtilis (NRRL B 4378) and Enterococcus faecium (NRRL-B 2354) were obtained from Agricultural Research Service Culture Collection (USA). Staphylococcus aureus (ATCC 29213), Staphylococcus epidermidis (ATCC 12228) were obtained from ATCC.

\subsection{Strains and Preparation of Stock Cultures}

Some of the strains were purchased as lyophilized powders from suppliers and they were inoculated into appropriate broths and incubated overnight in optimum conditions (time, temperature, shaking, etc.). In order to prolong usage time, stock cultures and their reserves were prepared in $40 \%$ glycerol broths by inoculation of the fresh culture (1:1). Stock cultures were labeled and kept in the $-80{ }^{\circ} \mathrm{C}$ for further studies.

\subsection{Determination of the Microbial Load in Assays}

In this study in order to adjust the microbial load, the incubation and inoculation parameters and the procedures were kept same and microbial loads were confirmed each time by fixing the OD (optical density) values of cultures inoculated into 96 well plates corresponding to certain numbers of bacteria $(\mathrm{CFU} / \mathrm{ml})$ that were determined by colony counting method.

\subsubsection{Minimum Inhibition Concentration of Plant Extract by Micro-dilution Assay}

\subsection{Determination of the MIC Values of Plant Extracts}

In order to determine the minimum inhibition concentrations (MIC) of the plant extracts; a gram negative (E. coli) and two gram positive bacteria ( $S$. aureus and $S$. epidermidis) were used. A serial 2-fold micro-broth dilution method [9] was performed to determine the MICs of plant extract. The crude plant extract was dissolved in dimethylsulfoxide (DMSO) as a stock solution having a concentration of $100 \mathrm{mg} / \mathrm{ml}$. Two fold serial dilutions of the extract solution were carried out, with a final concentration of $50 \mathrm{mg} / \mathrm{ml}$ by using sterile deionized water. Then $100 \mu l$ of each extract concentration and $95 \mu l$ nutrient broth were added to each well of 96 well microplate and each well inoculated with $5 \mu \mathrm{l}$ of $6 \mathrm{~h}$ incubated bacterial suspensions after standardized by adjusting their optical densities at $420 \mathrm{~nm}$ by UV spectrophotometer (Perkin Elmer) to obtain 0.8- 1.2 absorbance corresponding to approximately $107 \mathrm{CFU} / \mathrm{ml}$. Negative and positive controls were also carried out for each strain. Negative controls were performed by serial dilutions of DMSO (50\%) and the other negative control well consisted of $195 \mu$ of NB (Nutrient broth) and $5 \mu \mathrm{l}$ of the standard inoculum. Positive control wells consisted of serial dilutions of penicillin (400U) and gentamicin $(10 \mathrm{mg} / \mathrm{ml})$ antibiotics.

The assay plates were incubated at $37{ }^{\circ} \mathrm{C}$ for $24 \mathrm{~h}$ and the growth kinetic assays for each strain were performed by duplicate growth curves and observed as turbidity determined by a 
microplate reader (Varioskan) at $620 \mathrm{~nm}$. MIC results for extracts were reported as $\mathrm{mg} / \mathrm{mL}$. These spectrophotometric measurements of MIC values were carried out with a standardized protocol of Varioskan multiplate reader. After the $24 \mathrm{~h}$ incubation, INT was added into each test well in order to ensure visible indication of minimum inhibition concentrations. INT reacts with the metabolites produced by the microorganisms and the wells with the microorganism turn to pink color.

\subsubsection{GC Analysis of Cistus Plant Extract}

After extraction, each sample was analyzed by GC equipment (Agilent 7890A) with a FID. The samples $(20 \mu \mathrm{l})$ were injected into the injection port. A capillary column HP-5 (30m x $320 \mu \mathrm{m} \times 0.25 \mu \mathrm{m}$ film thickness) (Agilent) was used for chromatographic separation. The used temperature program was $5 \mathrm{~min}$ at $50{ }^{\circ} \mathrm{C}$ isothermal and an increase of $5{ }^{\circ} \mathrm{C} / \mathrm{min}$ to 200 ${ }^{0} \mathrm{C}$. Helium was used at $2 \mathrm{ml} / \mathrm{min}$ as the carrier gas. The temperature of injector and detector was 250 and $270{ }^{\circ} \mathrm{C}$, respectively.

\subsubsection{FT-IR Analysis of Cistus Plant}

The obtained gum and resin from Cistus plant was further investigated by using FT-IR analysis and the resulting spectrum was studied in order to understand the functional groups present. The gum and resin samples were dried and then the infrared spectra were obtained. The spectra of the samples were recorded in the $4000-650 \mathrm{~cm}^{-1}$ region at room temperature.

\section{RESULTS and DISCUSSIONS}

\subsection{Yield of Cistus Plant Extraction}

Extraction yield of Cistus plant was found as $19.05 \%$. After the extraction, the precipitate of Labdanum gum was obtained with the naturalization of the aqueous extract solution. The yield of the obtained gum was $1 \%$.

\subsection{Determination of Total Phenol Content and Antioxidant Capacity}

Phenolic compounds are a class of antioxidant agents which act as free radical terminators [10]. Phenolic compounds inhibit lipid oxidation by scavenging free radicals, chelating metals, activating antioxidant enzymes, reducing tocopherol radicals and inhibiting enzymes that cause oxidation reactions [11]. Total phenol contents of Cistus plant were analyzed with the FolinCiocalteu reagent. Total phenolic content of Cistus extract was determined as $520 \pm 15 \mathrm{mg}$ GAE/ g extract. By using a single system on photochem which was determined by Popov and Lewin [7] and commercialized by Analytik Jena AG (Jena Germany) antioxidant capacity of water soluble (ACW) and antioxidant capacity of lipid soluble (ACL) compounds were detected. Determination of water soluble and lipid soluble antioxidant capacities separately in an extract allows us to evaluate the plant material accurately. ACW of Cistus extract was found as $650 \pm 80 \mu \mathrm{g}$ Ascorbic acid/ mg extract and ACL of Cistus extract was found as $540 \pm 30 \mu \mathrm{g}$ Trolox Equivalent/ mg extract. Differences in the antioxidant activities in the ACW and ACL test results were expected due to the variations in concentrations of the lipid and water soluble antioxidant groups in the same plant extract

\subsection{Antibacterial Activities of Plant Extract}

\subsubsection{Disc Diffusion Assays}

In the disc diffusion assays, three bacteria species (E.coli (NRRL B- 3008), Bacillus subtilis (NRRL B 4378) and Enterococcus faecium (NRRL-B 2354)) were chosen to determine the antimicrobial activities of plant extract. E. coli is a gram negative bacterium while the other two species are gram positive bacteria. The extract of Cistus leaves was more effective against 
gram-positive bacteria than gram-negative bacteria, depending on the different structural and inherited features of these two groups of bacteria (Table 1).

Table 1. Results for disc diffusion of plant extract (expressed in $\mathrm{mm}$ )

\begin{tabular}{ccccc}
\hline Plant species & Plant part & E.coli & E.faecium & B.subtilis \\
\hline Cistus genus & Leaf & $7.56 \pm 0.556$ & $9.59 \pm 0.586$ & $9.19 \pm 0.155$ \\
\hline
\end{tabular}

\subsubsection{MIC (Minimum Inhibition Concentration) Assays}

MIC and disc diffusion assay results were given in Table 2. As seen in the results extract of Cistus sp. as effective as the positive control antibiotics. Comparing the MIC results between gram positive and gram negative bacteria Cistus extract was more effective on gram positive bacteria at relatively lower concentrations.

Table 2. MIC and disc diffusion results of plant extract and controls.

\begin{tabular}{lllllll}
\hline \multirow{3}{*}{ Plant species } & $\begin{array}{l}\text { E. coli } \\
\text { Disc } \\
\text { diff.(mm) }\end{array}$ & $\begin{array}{l}\text { MIC } \\
(\mathrm{mg} / \mathrm{ml})\end{array}$ & $\begin{array}{l}\text { S.aureus } \\
\text { Disc } \\
\text { diff.(mm) }\end{array}$ & $\begin{array}{l}\text { MIC } \\
(\mathrm{mg} / \mathrm{ml})\end{array}$ & $\begin{array}{l}\text { S.epidermidis } \\
\text { Disc } \\
\text { diff.(mm) }\end{array}$ & $\begin{array}{l}\text { MIC } \\
(\mathrm{mg} / \mathrm{ml})\end{array}$ \\
\hline Cistus genus & 7.56 & 25 & 7.84 & 1.56 & 7.34 & 0.78 \\
Positive controls & Disc & MIC & Disc & MIC & Disc & MIC \\
Penicillin & diff.(mm) & $(\mathrm{mg} / \mathrm{ml})$ & diff.(mm) & $(\mathrm{mg} / \mathrm{ml})$ & $\begin{array}{l}\text { diff.(mm) } \\
(\mathrm{mg} / \mathrm{ml})\end{array}$ \\
Gentamicin $(\mu \mathrm{g} / \mathrm{ml})$ & 7.02 & 2 & $» 25$ & $>0.02$ & $» 25$ & $>0.02$ \\
\hline
\end{tabular}

Minimum inhibitory concentration (MIC) is the lowest concentration at which an antimicrobial substance will inhibit a particular microbial growth under specified test conditions. The MIC measured for an antimicrobial compound is the lowest concentration that will prevent growth; it does not necessarily mean that the organisms in the test have been killed, it could just stop the growing (increasing in size or number) [12]. In Figure 1 antimicrobial effect of Cistus extract on E.coli with different concentrations were given.

$\mathrm{A}$ is the negative control which has no plant extract, has a pinkish color. As seen in Figure 1 from A to $\mathrm{H}$ by diluting the concentration of Cistus extract, pinkish color in the well of microplate was significant which showed more similarity with the negative control (growth with no extract solution). By diluting the Cistus extract in the wells of microplate antimicrobial effect of the plant extract was decreased. When the results were compared with negative control in well labeled as A pinkish color observed in the lowest concentration can be taken as MIC value of Cistus extract. After the results were evaluated MIC value of Cistus extract was found as $25 \mathrm{mg} / \mathrm{ml}$. 


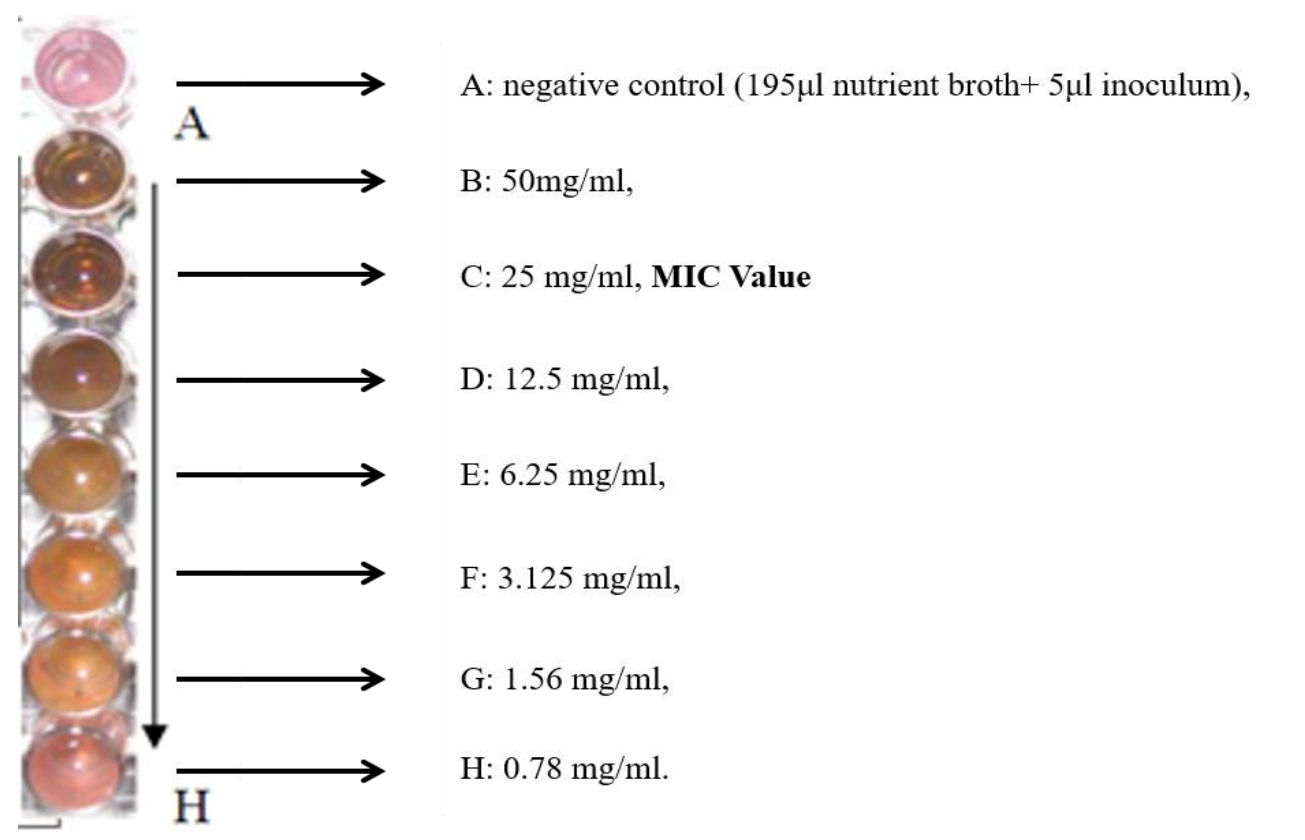

Figure 1. MIC value of the extract of Cistus leaves for E. coli

\subsection{FT-IR Analysis}

In Figure 2 FT-IR spectra of labdanum gum and resin were given.

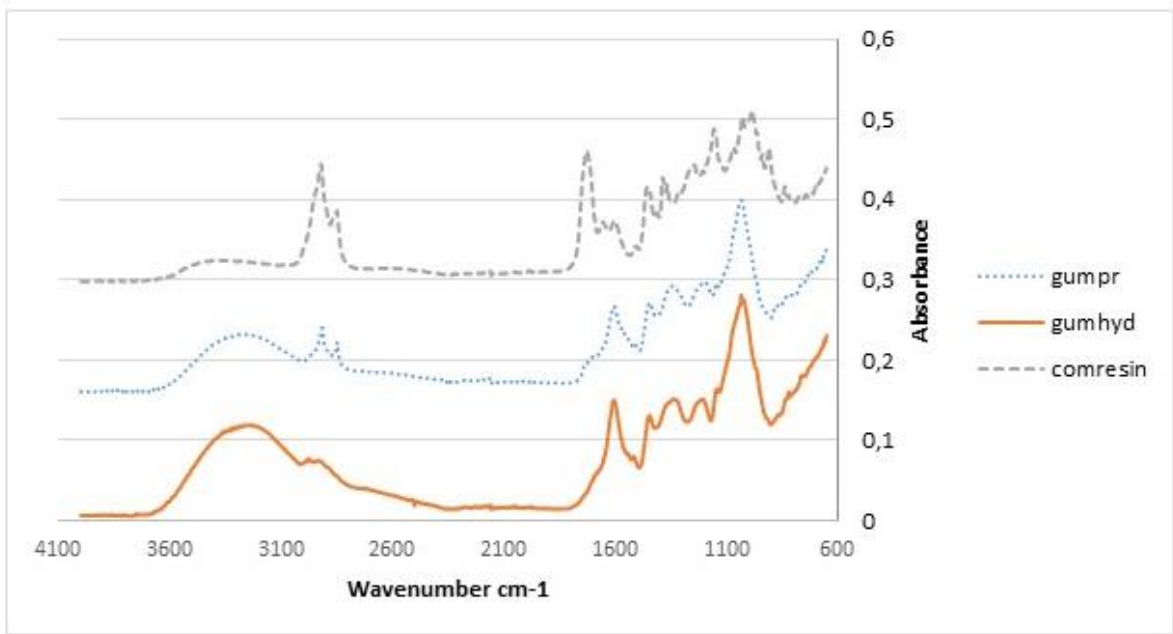

Figure 2. FTIR Spectra of Resin and Gum

FTIR spectrum of the sample showed $-\mathrm{OH},-\mathrm{C}=\mathrm{O}, \mathrm{C}-\mathrm{H}, \mathrm{C}-\mathrm{O}-\mathrm{C}, \mathrm{C}-\mathrm{N}$ and $\mathrm{C}=\mathrm{C}$ absorptions characteristics of resin and gum from Cistus sp. The vibrational band associated with carbohydrates is predominant in $1030-1072 \mathrm{~cm}^{-1}$ spectral regions (C-O-C stretch). The Cistus resinoid and gum bands at 1443, 1203 and $837 \mathrm{~cm}^{-1}$ can be associated with the vibrations of aromatic rings. The $\mathrm{C}-\mathrm{H}$ bond (where the hydrogen is attached to a carbon which is singlybonded to everything else) absorbs somewhere in the range from $2800-3000 \mathrm{~cm}-1$. The carbonoxygen double bond, $\mathrm{C}=\mathrm{O}$, is one of the really useful absorptions, found in the range 1680 $1820 \mathrm{~cm}^{-1}$. The other really useful bond is the O-H bond. It is easily recognized in an acid because it produces a very broad trough in the range $3200-3300 \mathrm{~cm}^{-1}$. Absorbance range for the functional groups was summarized in Table 3. 
Table 3. Absorbance range

\begin{tabular}{cc}
\hline Functional group & Wavenumber $\left(\mathrm{cm}^{-1}\right)$ \\
\hline OH & $3200-3300$ \\
C-H (stretch) & $2800-3000$ \\
C=C & $1600-1650$ \\
C=O & $1680-1820$ \\
C-O-C & $1030-1072$ \\
C-N & $1180-1360$ \\
\hline
\end{tabular}

\subsection{GC Analysis of Cistus Extract}

From the literature survey, it was known that Cistus sp. the volatile components is an extremely complex mixture of aldehydes, alkanes, fatty acids, norisoprenoids, oxygenated monoterpenes, sesquiterpene hydrocarbons, oxygenated sesquiterpenes, diterpene, and aromatics. Quantitatively, the major fraction of the oil was given by fatty acids $(67.8 \%)$ which were mainly represented by linolenic, hexadecanoic and linoleic acids. The second group of volatiles was represented by alkanes $(13.5 \%)$ with n-pentacosane, n-heptacosane and ntricosane as the most abundant compounds. These components were also found in the volatile oil from C.creticus growing in Turkey. The third most abundant group of volatiles was represented by aldehydes with n-pentadecanal, n-tridecanal and n-tetradecanal as the most abundant. These acyclic aldehydes are the oxidation products of fatty acids or glyceridly esters occurring during hydrodistillation. Terpenoids gave a minor contribution to the oil (6.9\%) [13].

The most abundant compounds were $\alpha$-pinene, o-guaiacol, camphor and camphene. In addition to that limonene, bornyl acetate, $p$-cymene, $\beta$-pinene were identified as other volatiles in the volatile fraction of Cistus sp. [14]. It is also known from the literature survey that ethanol extract of Cistus sp. has a cytotoxic activity against human cancer cells [15].

Most studies on the volatile fraction of Cistus sp. are focused on the essential oil obtained either by steam distillation or hydrodistillation as well as other common extraction techniques involving the use of solvents such as hexane. In our study solvent extract of labdanum resin was analyzed with GC. The presence of a trace amount of volatile compounds in labdanum resin indicated the possible antimicrobial activity of labdanum resin. In Figure 3, Chromatogram of the hexane extract of labdanum resin was given.

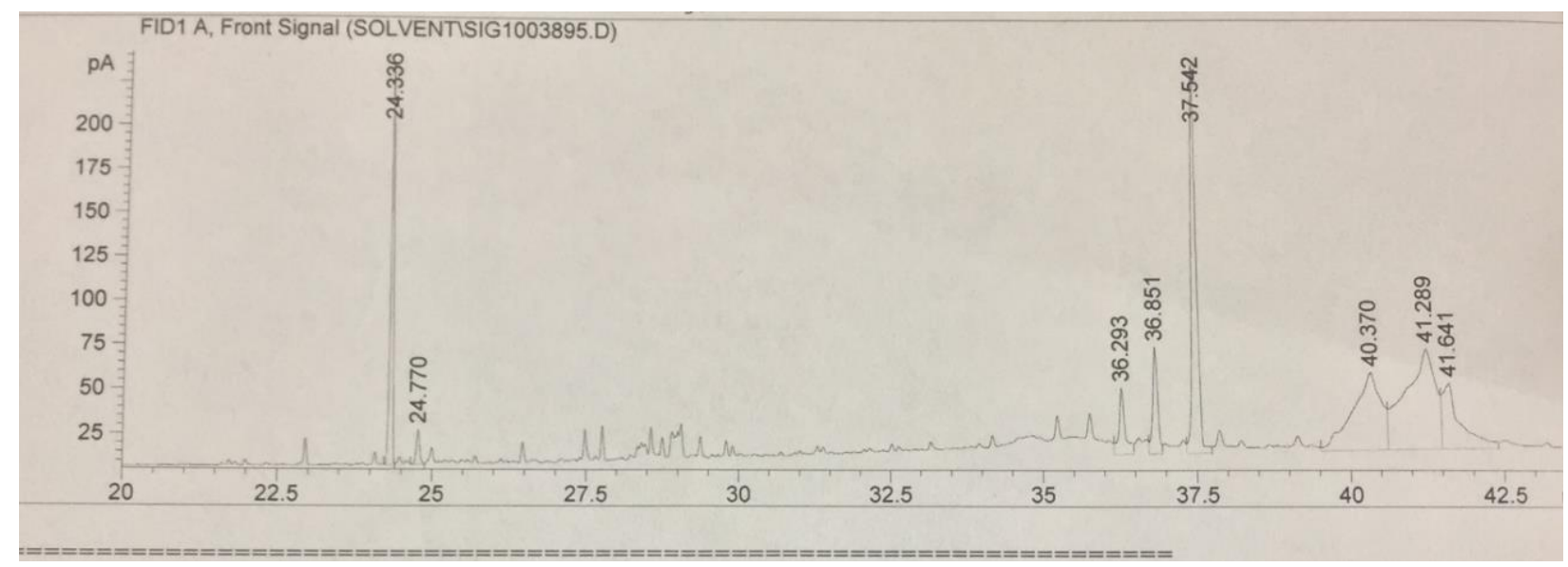

Figure 3. Chromatogram of the Hexane Extract of Labdanum Resin

Although not identified these compounds could possibly be limonene, p-cymene, bornyl acetate and E-caryophyllene which are consistent with the data from the literature survey. In Figure 4, Chromatogram of the ethanol extract of labdanum resin was given. 


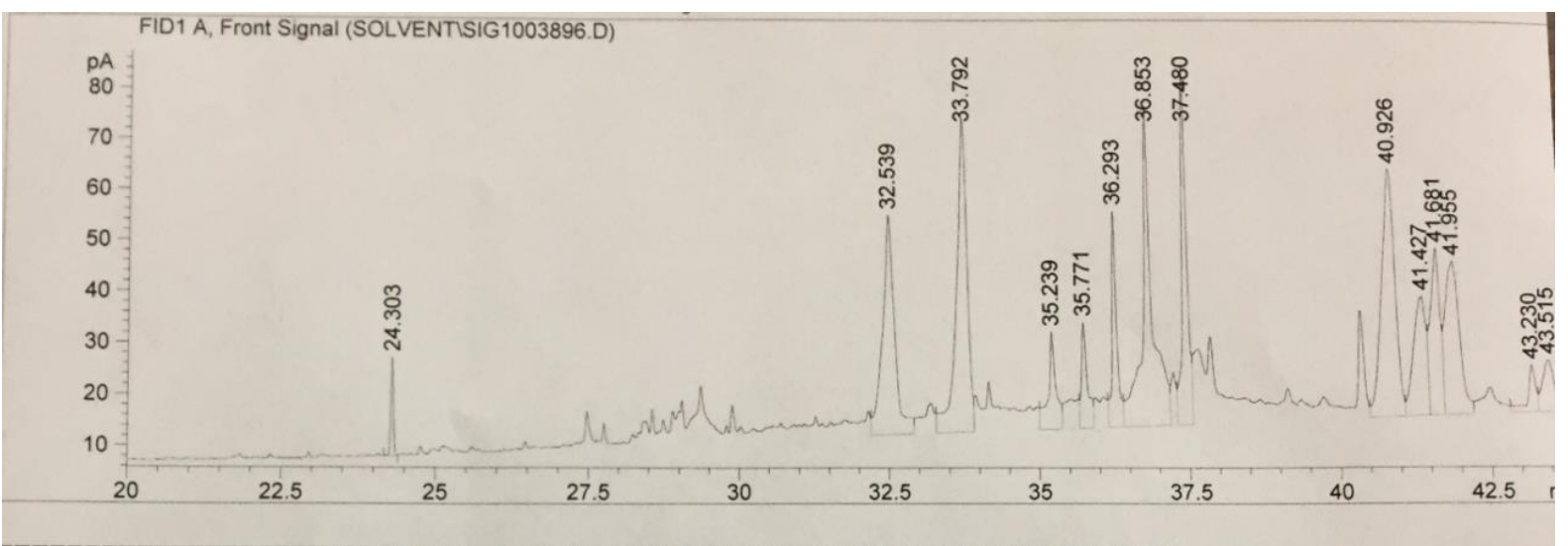

Figure 4. Chromatogram of the Ethanol Extract of Labdanum Resin

As seen from the figures there are differences between Figure 3 and 4. These different peaks representing different compounds may be due to the polarity difference of the used solvent. In addition to the GC analysis of labdanum resin, GC analysis of labdanum essential oil was performed. In Figure 5 the GC chromatogram of the essential oil in hexane was given. By comparing the Figure 3 and Figure 5 it is possible to say labdanum resin and labdanum essential oil has different components as expected. From the studies done on Cistus essential oil, the main components of the essential oil were identified as 13-epi-manoyl oxide (18.5\%), manool (7.2\%), labda-7, 14-dien-13-ol (3.8\%), manoyl oxide (4.2\%) and slareol (2.7\%) [16].

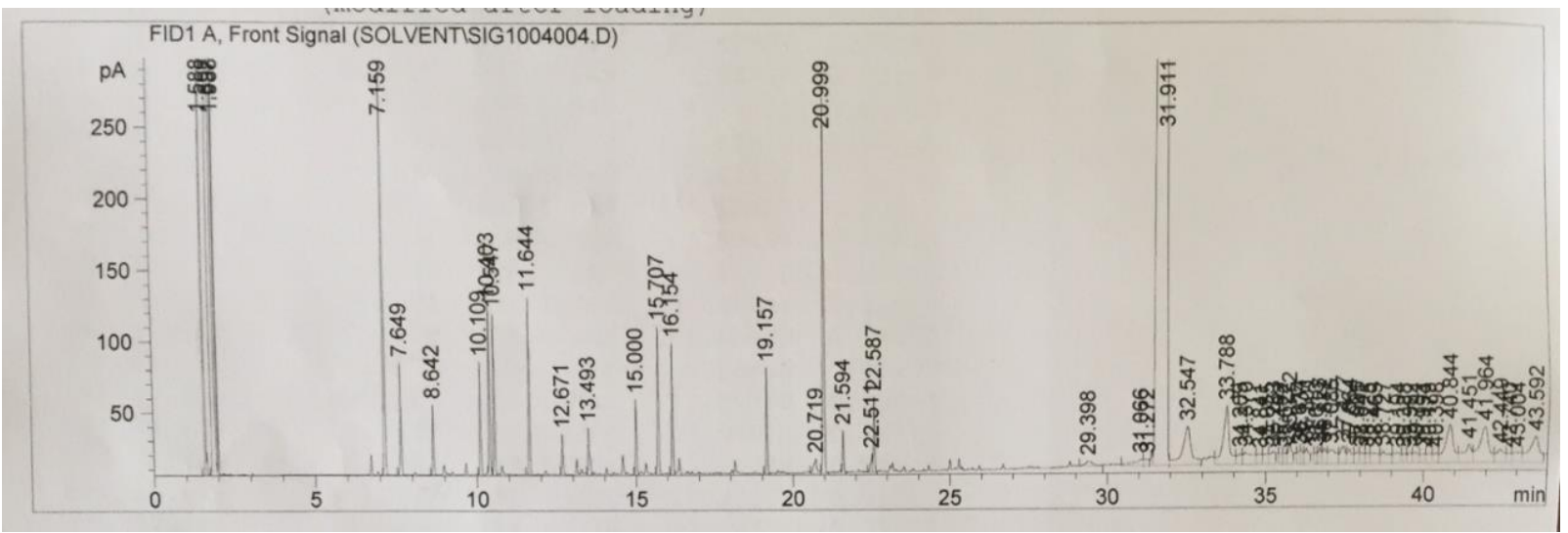

Figure 5. Chromatogram of the Labdanum Essential Oil in Hexane

In the Figure 6 chromatogram of the labdanum essential oil in ethanol was given. Same as the labdanum resin figures, there are differences between Figure 5 and 6 . These different peaks may be as a result of the polarity difference of the used solvent.

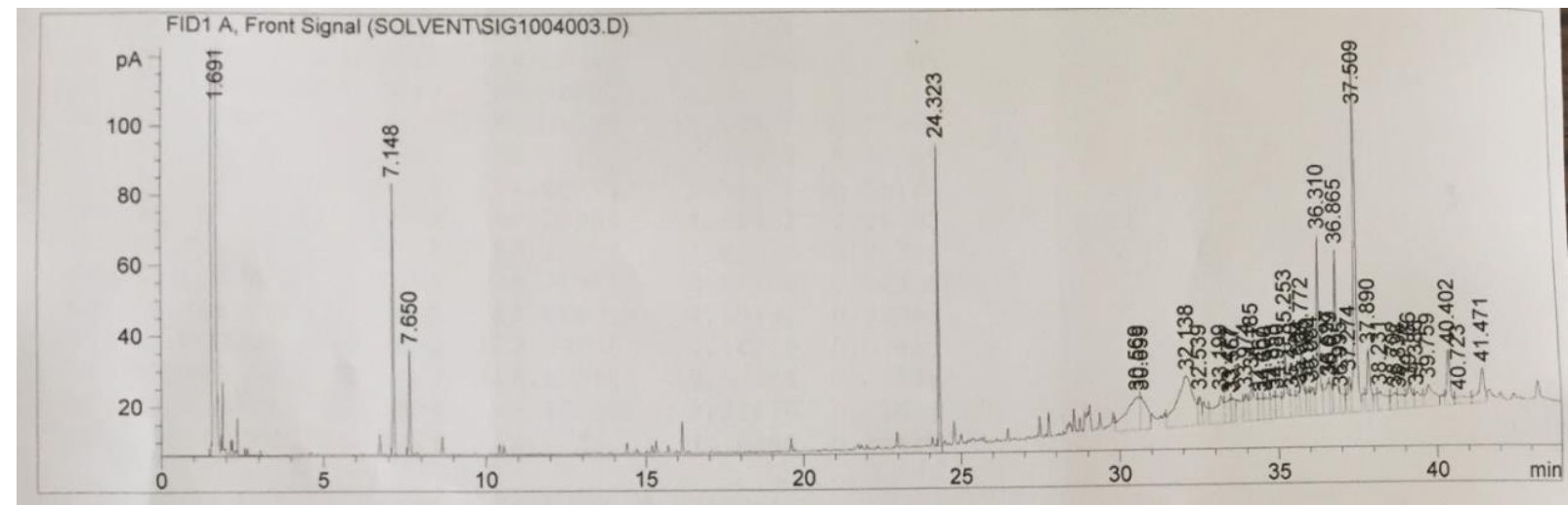

Figure 6. Chromatogram of the Labdanum Essential Oil in Ethanol 


\section{CONCLUSION}

In The leaves of Cistus sp. collected from Urla region was used to prepare extract with antioxidant capacities and antimicrobial activities. Cistus extract was as effective as antibiotics used as a positive control. These results revealed that extract of Cistus leaves could be promising to be used as the natural antibacterial agent. After obtaining the essential oil of the leaves with hydrodistillation the prepared gum still had several volatiles confirmed by GC analysis. With these results, it is possible to use Cistus $s p$. as a natural antimicrobial source for the production of different types of products, including essential oil, gum, resinoid and extract.

\section{Acknowledgement}

Authors acknowledge NPRO Consult. Nat. Prod. Biotech. Ltd. Co. and Small \& Medium Enterprises Development Organization (KOSGEB) for their supports.

\section{Conflict of Interests}

Authors declare that there is no conflict of interests.

\section{REFERENCES}

[1]. Lubbe, A., \& Verpoorte, R. (2011). Cultivation of medicinal and aromatic plants for specialty industrial materials. Industrial Crops and Products, 34(1), 785-801.

[2]. Rios, J. L., \& Recio, M. C. (2005). Medicinal plants and antimicrobial activity. Journal of ethnopharmacology, 100(1), 80-84.

[3]. Atlas, R. M., Brown, A. E., \& Parks, L. C. (1995). Laboratory manual of experimental microbiology. Mosby.

[4]. Cetin, H., \& Yanikoglu, A. (2006). A study of the larvicidal activity of Origanum (Labiatae) species from southwest Turkey. Journal of Vector ecology, 31(1), 118-122.

[5]. Vertuani, S., Braccioli, E., Buzzoni, V., \& Manfredini, S. (2002). Antioxidant capacity of Adansonia digitata fruit pulp and leaves. Acta phytotherapeutica, 2(5), 2-7.

[6]. Barros, L., Dueñas, M., Alves, C. T., Silva, S., Henriques, M., Santos-Buelga, C., \& Ferreira, I. C. (2013). Antifungal activity and detailed chemical characterization of Cistus ladanifer phenolic extracts. Industrial Crops and Products, 41, 41-45.

[7]. Popov, I., \& Lewin, G. (1999). Antioxidative homeostasis: characterization by means of chemiluminescent technique. Methods in enzymology, 300, 437-456.

[8]. Singleton, V. L. (1999). Analysis of total phenols and other oxidation substrates and their antioxidants by means of Folin-Ciocalteu reagent. Meth. Enzymol., 299, 152-178.

[9]. Kuete, V., Metuno, R., Ngameni, B., Tsafack, A. M., Ngandeu, F., Fotso, G. W., \& Beng, V. P. (2007). Antimicrobial activity of the methanolic extracts and compounds from Treculia obovoidea (Moraceae). Journal of ethnopharmacology, 112(3), 531-536.

[10].Pourmorad, F., Hosseinimehr, S. J., \& Shahabimajd, N. (2006). Antioxidant activity, phenol and flavonoid contents of some selected Iranian medicinal plants. African journal of biotechnology, 5(11).

[11]. Harrison, K., \& Were, L. M. (2007). Effect of gamma irradiation on total phenolic content yield and antioxidant capacity of almond skin extracts. Food Chemistry, 102(3), 932-937.

[12] Zakaria, Z., Sreenivasan, S., \& Mohamad, M. (2007). Antimicrobial Activity of Piper ribesoides Root Extract Against Staphylococcus aureus. Journal of Applied Biological Sciences, 1(3). 
[13].Maggi, F., Lucarini, D., Papa, F., Peron, G., \& Dall'Acqua, S. (2016). Phytochemical analysis of the labdanum-poor Cistus creticus subsp. eriocephalus (Viv.) Greuter et Burdet growing in central Italy. Biochemical Systematics and Ecology, 66, 50-57.

[14].Morales-Soto, A., Oruna-Concha, M. J., Elmore, J. S., Barrajón-Catalán, E., Micol, V., Roldán, C., \& Segura-Carretero, A. (2015). Volatile profile of Spanish Cistus plants as sources of antimicrobials for industrial applications. Industrial Crops and Products, 74, 425-433.

[15].Skorić, M., Todorović, S., Gligorijević, N., Janković, R., Živković, S., Ristić, M., \& Radulović, S. (2012). Cytotoxic activity of ethanol extracts of in vitro grown Cistus creticus subsp. creticus L. on human cancer cell lines. Industrial Crops and Products, 38, 153-159.

[16].Paolini, J., Falchi, A., Quilichini, Y., Desjobert, J. M., De Cian, M. C., Varesi, L., \& Costa, J. (2009). Morphological, chemical and genetic differentiation of two subspecies of Cistus creticus L. (C. creticus subsp. eriocephalus and C. creticus subsp. corsicus). Phytochemistry, 70(9), 1146-1160. 